\title{
Synthetic Aperture Hitchhiker Imaging using Non-Cooperative Sources of Opportunity
}

\author{
Can Evren Yarman, Birsen Yazıcı and Margaret Cheney \\ Rensselaer Polytechnic Institute, 110 Eighth Street, Troy, NY
}

\begin{abstract}
A hitchhiker is a passive radar receiver that relies on sources of opportunity to perform radar tasks. ${ }^{1-4}$ In this paper, we consider a synthetic-aperture radar (SAR) system with static non-cooperative transmitters and mobile receivers traversing arbitrary trajectories and present an analytic image formation method. Due to its combined synthetic aperture and hitchhiking structure, we refer to the system under consideration as synthetic aperture hitchhiker (SAH). Our approach is applicable to cooperative and/or non-cooperative and static and/or mobile sources of opportunity.

Conventional SAR processing involves correlation of the received signal from a receiver with the transmitted waveform as a first step of the image formation. For passive SAR, however, the transmitted waveform is not necessarily known. Instead, we use spatio-temporal correlation of received signals. Given a pair of receivers, the spatio-temporal correlation method compares the received signals to identify a target within the illuminated scene. We combine this with microlocal techniques to develop a filtered backprojection (FBP) type inversion method for passive SAR. ${ }^{5}$

Combined correlation-FBP inversion method does not require the knowledge of the transmitter locations. Furthermore, FBP inversion has the advantage of computational efficiency and image formation under non-ideal conditions, such as arbitrary flight trajectories and non-flat topography.
\end{abstract}

\section{INTRODUCTION}

With the rapid growth in the number of TV and radio broadcasting stations,${ }^{6-10}$ mobile phone base stations, ${ }^{11,12}$ and terrestrial and space based communication and navigation satellites, ${ }^{13-18}$ hitchhikers offer a viable approach to urban and rural surveillance radar as stand-alone or adjunct to active radar systems.

Due to their receive only structure, the hitchhikers do not radiate, therefore they do not have environmental impact, and are far less vulnerable than transmitters to electronic counter measures. Furthermore, hitchhikers offer the following advantages. They are small and easy to install, and can be mounted on ground based mobile platforms. They can be rapidly deployed. Their manufacturing and operation costs are low. They require low powers for operation since they rely on other sources. They can be employed to customize an application specific coverage area. Finally, since the stealth technology is designed primarily for monostatic radar, hitchhikers has a potential to counter stealth technology. $2,14,19,20$

To our knowledge, existing passive radar systems ${ }^{7,9-11,14-16,21}$ consist of multiple receiver antennas. For each possible transmitter there is at least one designated receiver that has a direct line of sight to the

Further author information: (Send correspondence to C.E.Y.) C.E.Y.: E-mail: yarman@ecse.rpi.edu, Telephone: +1 (518) 2766085

B.Y.: E-mail: yazici@ecse.rpi.edu, Telephone: +1 (518) 276 2905, Fax: +1 (518) 2766261

M.C.: E-mail: cheney@rpi.edu, Telephone: +1 (518) 276 2646, Fax: +1 (518) 2764824

Algorithms for Synthetic Aperture Radar Imagery XIV, edited by Edmund G. Zelnio, Frederick D. Garber, Proc. of SPIE Vol. 6568, 656806, (2007) · 0277-786X/07/\$18 · doi: 10.1117/12.719660 
transmitter that can receive target free reference signal. Target detection is performed by correlating the reference signal with the signals obtained from the other receivers. While these approaches provide a novel way to perform detection without the knowledge of the transmitted signal, they require knowledge of the number of transmitters, their locations along with receiver antennas with high directivity in order to isolate the direct path from other paths.

In the case of two or more receivers, an alternative approach is to correlate the received signals obtained at different receiver locations, which we will refer to as spatio-temporal correlation, and look at the relative change in the correlated signals to perform target detection. ${ }^{22-24}$ Unlike the approaches in, ${ }^{7,9-11,14-16,21}$ this approach does not require any knowledge about the transmitters.

In this paper, we consider a synthetic-aperture imaging system consisting of receivers with poor directivity traversing arbitrary flight trajectories that use sources of opportunity for imaging as illustrated in Figure 1, and provide a spatio-temporal correlation-based imaging method to recover the target scene radiance. The advantage of our method compared to the methods used in the aforementioned passive radar imaging techniques is that it can be employed for passive imaging in the presence of both cooperative and non-cooperative sources of opportunity without requiring direct path to each transmitter for reference signal extraction and thus receivers with high directivity. The method can be easily extended to multistatic synthetic aperture hitchhikers.

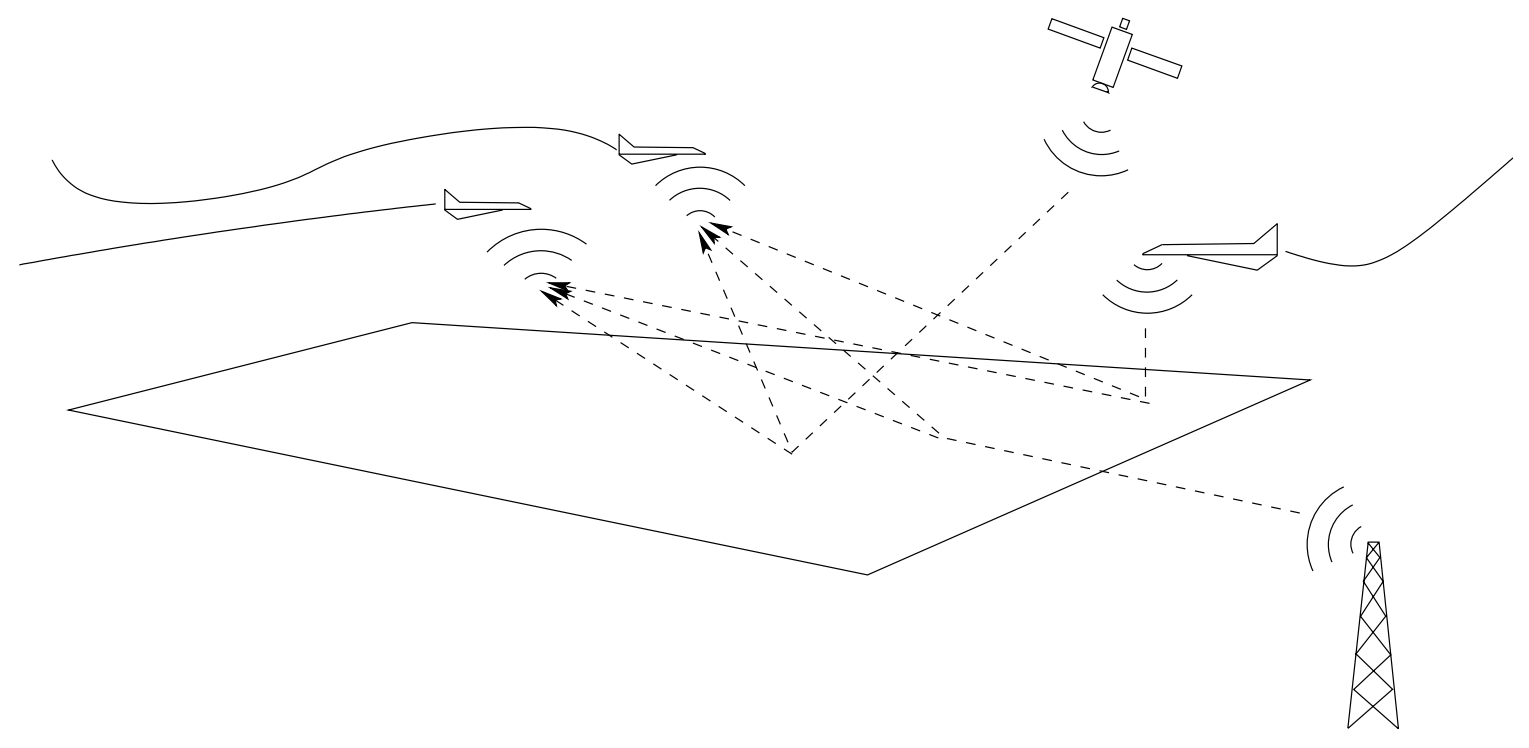

Figure 1. Synthetic aperture hitchhiker geometry.

In the optic, acoustic and seismic literature, analog of the spatio-temporal correlation method have been successfully applied to imaging under the context of coherent-interferometry, time-reversal and phase conjugation ( $\mathrm{see}^{25-27}$ and references therein for a review and recent advances on coherentinterferometry, time-reversal and phase conjugation).

Our treatment combines the spatio-temporal correlation methods presented in ${ }^{23,28}$ with microlocal techniques $^{29,30}$ to develop FBP-type reconstruction methods for SAH. Given a pair of receivers, the spatio-temporal correlation method correlates received signals from different receivers to identify a target within the illuminated scene, eliminating the need for knowledge about the transmitter location and waveform. Microlocal techniques provide an approximate FBP-type inversion method; however, if an exact inversion is possible, the result often reduces to the exact inversion formula. Furthermore, 
the FBP-type inversions have the desirable property that visible edges in the target scene appear at the right location and at the right orientation. Thus, we perform reconstruction in three steps: First correlate the received signals; next filter the correlated signal; and finally backproject the correlated and filtered signals along isorange contours (see Section 3 for definition). We present the performance of the method in numerical simulations.

While for the current discussion, we consider static sources of opportunity, the method we introduce is also applicable to mobile sources of opportunity.

The organization of the paper is as follows: In Section 2, we introduce the forward model. In Section 3, we present the image reconstruction method. In Section 4, we demonstrate the performance of the method in numerical simulations. Finally, we conclude our discussion in Section 5 .

\section{FORWARD MODEL}

Let $\gamma_{R_{1}}(s)$ and $\gamma_{R_{2}}(s), s \in \mathbb{R}$ be the SAH trajectories, as shown in Figure 1 . Let $\mathbf{x}=(\boldsymbol{x}, \psi(\boldsymbol{x})) \in \mathbb{R}^{3}$ denote the surface of the earth, where $\boldsymbol{x}=\left(x_{1}, x_{2}\right)$ and $\psi: \mathbb{R}^{2} \rightarrow \mathbb{R}$ is a known smooth function.

We assume that the electromagnetic waves propagate in free-space and then scatter in a thin region at the earth's surface. Under the start-stop approximation, the single-scattering (Born) approximation of the contribution to the received signal at the $i^{\text {th }}(i=1,2)$ receiver due to a transmitter located at $\mathbf{y} \in \mathbb{R}^{3}$ can be modeled as: ${ }^{29}$

$$
d_{i, \mathbf{y}}(s, t) \approx \int \mathrm{e}^{-\mathrm{i} 2 \pi \omega\left(t-r_{i, \mathbf{y}}(s, \boldsymbol{x}) / c_{0}\right)} A_{R_{i}}(\omega, s, \boldsymbol{x}) A_{T}(\omega, \boldsymbol{x}, \mathbf{y}) G(\boldsymbol{x}) d \omega d \boldsymbol{x},
$$

where $\mathrm{i}=\sqrt{-1}, t$ is the fast-time, $s$ is the slow-time variable that parameterizes the trajectory, $c_{0}$ denotes the speed of light, $r_{i, \mathbf{y}}(s, \mathbf{x}) / c_{0}$, where $r_{i, \mathbf{y}}=|\mathbf{y}-\mathbf{x}|+\left|\mathbf{x}-\gamma_{R_{i}}(s)\right|$, is the total travel time, $G(\boldsymbol{x})$ denotes the ground reflectivity, and

$$
\begin{aligned}
& A_{R_{i}}(\omega, s, \boldsymbol{x})=\sqrt{\frac{\pi}{2}} \frac{\omega J_{R_{i}}(\omega, s, \boldsymbol{x})}{\left|\gamma_{R_{i}}(s)-\mathbf{x}\right|} \\
& A_{T}(\omega, \boldsymbol{x}, \mathbf{y})=\sqrt{\frac{\pi}{2}} \frac{\omega J_{T}(\omega, \boldsymbol{x}, \mathbf{y})}{|\mathbf{x}-\mathbf{y}|}
\end{aligned}
$$

In (2) $J_{R_{i}}$ is the $i^{\text {th }}$ receiver antenna beam pattern and $J_{T}$ is the transmitter antenna beam pattern located at $\mathbf{y}$ (which also includes the transmitter waveform).

The received signal at the $i^{\text {th }}$ receiver is given by the superposition of (1) over all transmitters:

$$
d_{i}(s, t)=\int d_{i, \mathbf{y}}(s, t) d \mathbf{y}
$$

The ideal image reconstruction problem is to estimate $G$ from the knowledge of $d_{i}(s, t), i=1,2$, for some range $\left[s_{a}, s_{b}\right]$ and $\left[0, t_{0}\right]$ of $s$ and $t$, respectively. For monostatic SAR and BISAR, the general strategy for estimating $G$ is to perform matched-filtering followed by filtered backprojection (MFFBP $).{ }^{29,31,32}$ MF-FBP introduced in, ${ }^{31,32}$ however, requires the received signal in (3) to be decomposed into the components due to each of the transmitters given in (1).

In the presence of non-cooperative sources of opportunity the received signal can not be decomposed into components due to each of the transmitters. In this case, we instead reconstruct the target scene 
radiance $R_{G}$ of the scene by filtered backprojecting the correlated received signals of different receivers. We refer to the resulting imaging method as correlation filtered backprojection (C-FBP).

We define the spatio-temporal correlation of $d_{1}$ and $d_{2}$ by

$$
d_{12}\left(s, s^{\prime}, t\right)=\int d_{1}(s, \tau) d_{2}^{*}\left(s+s^{\prime}, \tau-t\right) d \tau,
$$

where * denotes complex conjugation.

Considering that the sources of opportunity are non-cooperative, we treat the unknown terms transmitter antenna beam pattern $J_{T}$ and ground reflectivity $G$ statistically, and for ease of exposition, we also assume, without loss of generality, that $G$ and $J_{T}$ are zero mean random processes. Furthermore, we assume that $G$ and $J_{T}$ are statistically uncorrelated.

Let $C_{G}$ and $C_{T}$ denote the auto-covariances of $G$ and $J_{T}$, respectively:

$$
\begin{gathered}
C_{G}\left(\boldsymbol{x}, \boldsymbol{x}^{\prime}\right)=E\left[G(\boldsymbol{x}) G^{*}\left(\boldsymbol{x}^{\prime}\right)\right] \\
C_{J_{T}}\left(\omega, \boldsymbol{x}, \boldsymbol{x}^{\prime}, \mathbf{y}, \mathbf{y}^{\prime}\right)=E\left[J_{T}(\omega, \boldsymbol{x}, \mathbf{y}) J_{T}^{*}\left(\omega, \boldsymbol{x}^{\prime}, \mathbf{y}^{\prime}\right)\right]
\end{gathered}
$$

where $J_{T}^{*}$ denotes complex conjugate of $J_{T}$. Then

$$
\begin{aligned}
E\left[d_{12}\left(s, s^{\prime}, t\right)\right]=\int \mathrm{e}^{-\mathrm{i} 2 \pi \omega\left(t-\left[\left|\mathbf{x}-\boldsymbol{\gamma}_{R_{1}}(s)\right|-\left|\mathbf{x}^{\prime}-\boldsymbol{\gamma}_{R_{2}}\left(s+s^{\prime}\right)\right|\right] / c_{0}\right)} A_{R_{12}}\left(\omega, s, s^{\prime}, \boldsymbol{x}, \boldsymbol{x}^{\prime}\right) \\
\times\left[\int \mathrm{e}^{\mathrm{i} 2 \pi \omega\left(\left[|\mathbf{y}-\mathbf{x}|-\left|\mathbf{y}^{\prime}-\mathbf{x}^{\prime}\right|\right] / c_{0}\right)} C_{T}\left(\omega, \boldsymbol{x}, \boldsymbol{x}^{\prime}, \mathbf{y}, \mathbf{y}^{\prime}\right) d \mathbf{y} d \mathbf{y}^{\prime}\right] C_{G}\left(\boldsymbol{x}, \boldsymbol{x}^{\prime}\right) d \omega d \boldsymbol{x} d \boldsymbol{x}^{\prime},
\end{aligned}
$$

where

$$
A_{R_{12}}\left(\omega, s, s^{\prime}, \boldsymbol{x}, \boldsymbol{x}^{\prime}\right)=A_{R_{1}}(\omega, s, \boldsymbol{x}) A_{R_{2}}^{*}\left(\omega, s+s^{\prime}, \boldsymbol{x}^{\prime}\right)
$$

and

$$
C_{T}\left(\omega, \boldsymbol{x}, \boldsymbol{x}^{\prime}, \mathbf{y}, \mathbf{y}^{\prime}\right)=\frac{\pi}{2} \frac{\omega^{2} C_{J_{T}}\left(\omega, \boldsymbol{x}, \boldsymbol{x}^{\prime}, \mathbf{y}, \mathbf{y}^{\prime}\right)}{|\mathbf{x}-\mathbf{y}|\left|\mathbf{x}^{\prime}-\mathbf{y}^{\prime}\right|}
$$

Next, we make the incoherent-field approximation ${ }^{26}$ to (6) by assuming that $G$ and $J_{T}$ are uncorrelated in $\boldsymbol{x}$ and $\mathbf{y}$, respectively:

$$
\begin{gathered}
C_{G}\left(\boldsymbol{x}, \boldsymbol{x}^{\prime}\right)=R_{G}(\boldsymbol{x}) \delta\left(\boldsymbol{x}-\boldsymbol{x}^{\prime}\right) \\
C_{T}\left(\omega, \boldsymbol{x}, \boldsymbol{x}, \mathbf{y}, \mathbf{y}^{\prime}\right)=R_{T}(\omega, \boldsymbol{x}, \mathbf{y}) \delta\left(\mathbf{y}-\mathbf{y}^{\prime}\right) .
\end{gathered}
$$

$R_{G}$ is the electromagnetic radiation that is emitted from the surface of the target, and is referred to as the radiant exitance/emittance of the target or target radiance. ${ }^{26} R_{T}$ is the power of electromagnetic radiation emitted from the transmitter that is incident on the target surface, and is referred to as transmitter irradiance. ${ }^{26}$

Thus we simplify (6) to

$$
\begin{aligned}
E\left[d_{12}\right]\left(s, s^{\prime}, t\right) & =\mathcal{F}\left[R_{G}\right]\left(s, s^{\prime}, t\right) \\
& :=\int \mathrm{e}^{-\mathrm{i} 2 \pi \omega\left(t-r_{12}\left(s, s^{\prime}, \mathbf{x}\right) / c_{0}\right)} A_{R_{12}}\left(\omega, s, s^{\prime}, \boldsymbol{x}, \boldsymbol{x}\right) \tilde{R}_{T}(\omega, \boldsymbol{x}) R_{G}(\boldsymbol{x}) d \omega d \boldsymbol{x}
\end{aligned}
$$


where

$$
\tilde{R}_{T}(\omega, \boldsymbol{x})=\int R_{T}(\omega, \boldsymbol{x}, \mathbf{y}) d \mathbf{y}
$$

is the total transmitter irradiance and $r_{12}\left(s, s^{\prime}, \mathbf{x}\right)=\left|\mathbf{x}-\gamma_{R_{1}}(s)\right|-\left|\mathbf{x}-\gamma_{R_{2}}\left(s+s^{\prime}\right)\right|$. We will refer to $r_{12}$ as the hitchhiker range. Our aim is to reconstruct $R_{G}$ given $E\left[d_{12}\left(s, s^{\prime}, t\right)\right]$.

\section{IMAGE FORMATION}

We assumed that $G$ and $A_{T}$ are statistically uncorrelated in $\mathbf{x}$ and $\mathbf{y}$ and zero mean random processes. Additionally, we assume that for some $m, A_{12}=A_{R_{12}} \tilde{R}_{T}$ satisfies

$$
\sup _{(s, \boldsymbol{x}) \in K}\left|\partial_{\omega}^{\alpha} \partial_{s}^{\beta} \partial_{s^{\prime}}^{\beta^{\prime}} \partial_{x_{1}}^{\rho_{1}} \partial_{x_{2}}^{\rho_{2}} A_{12}\left(\omega, s, s^{\prime}, \boldsymbol{x}\right)\right| \leq C_{A}\left(1+\omega^{2}\right)^{\left(m_{A}-|\alpha|\right) / 2}
$$

where $K$ is any compact subset of $\mathbb{R} \times \mathbb{R}^{2}$, and the constant $C_{A}$ depends on $K, \alpha, \beta, \beta^{\prime}, \rho_{1}$, and $\rho_{2}$. In practice (12) is satisfied for transmitters and receivers sufficiently away from the illuminated region. This is the case especially for air-/space-borne transmitters and receivers, and broadcasting stations located on high grounds.

Furthermore, under the assumption (12), equation (10) defines $\mathcal{F}$ as a Fourier integral operator ${ }^{33}$ whose leading-order contribution comes from those points lying in the intersection of the illuminated surface and the hyperboloid $H_{12}\left(s, s^{\prime}, t\right)=\left\{\boldsymbol{x}: r_{12}\left(s, s^{\prime}, \mathbf{x}\right)=c_{0} t\right\}$. The curves formed by the intersection of the surface and $H_{12}\left(s, s^{\prime},.\right)$ will be referred to as isorange contours, at the slow-time parameters $s$ and $s^{\prime}$. For flat topography $\psi(\boldsymbol{x})=0$, the isorange contours are given by hyperbolas on the plane $x_{3}=0$. Thus an approximate inversion of $\mathcal{F}$ can be computed by a suitable backprojection of $d_{12}$ on to the isorange contours: ${ }^{33}$

$$
\mathcal{K}\left[\mathcal{F}\left[R_{G}\right]\right](\boldsymbol{z})=\int \mathrm{e}^{\mathrm{i} 2 \pi \omega\left(t-r_{12}\left(s, s^{\prime}, \mathbf{z}\right) / c_{0}\right)} Q\left(\boldsymbol{z}, \omega, s, s^{\prime}\right) \mathcal{F}\left[R_{G}\right]\left(s, s^{\prime}, t\right) d t d \omega d s d s^{\prime},
$$

where $\mathcal{K}$ will be referred to as the filtered backprojection operator with $Q$ being the filter which is chosen such that the leading order term of the point spread function of $\mathcal{L}=\mathcal{K} \mathcal{F}$ becomes Dirac delta function.

In this regard, we choose $Q$ to be ( $\sec ^{31}$ for the derivation of $Q$ )

$$
Q\left(\boldsymbol{z}, \omega, s, s^{\prime}\right)=\frac{\chi_{\Omega_{\boldsymbol{z}, s^{\prime}}}(\boldsymbol{\xi}(s, \omega))}{\eta\left(\boldsymbol{z}, \boldsymbol{z}, \boldsymbol{\xi}, s^{\prime}\right)} \frac{A_{12}^{*}\left(\omega, s, s^{\prime}, \boldsymbol{z}\right)}{\left|A_{12}\left(\omega, s, s^{\prime}, \boldsymbol{z}\right)\right|^{2}}
$$

where $\eta\left(\boldsymbol{x}, \boldsymbol{z}, \boldsymbol{\xi}, s^{\prime}\right)=|\partial(s, \omega) / \partial \boldsymbol{\xi}|$, is the determinant of the Jacobian that comes from the change of variables

$$
(s, \omega) \rightarrow \boldsymbol{\xi}=\frac{\omega}{c_{0}} D \psi(\boldsymbol{z}) \cdot\left[\frac{\mathbf{z}-\gamma_{R_{1}}(s)}{\left|\mathbf{z}-\gamma_{R_{1}}(s)\right|}-\frac{\mathbf{z}-\gamma_{R_{2}}\left(s+s^{\prime}\right)}{\left|\mathbf{z}-\gamma_{R_{2}}\left(s+s^{\prime}\right)\right|}\right],
$$

with

$$
D \psi(\boldsymbol{z})=\left[\begin{array}{ccc}
1 & 0 & \partial \psi(\boldsymbol{z}) / \partial z_{1} \\
0 & 1 & \partial \psi(\boldsymbol{z}) / \partial z_{2}
\end{array}\right]
$$


and $\chi_{\Omega_{z, s^{\prime}}}$ is a smooth cut-off function equal to one in most of the interior of $\Omega_{\boldsymbol{z}, s^{\prime}}$ and zero in the exterior of $\Omega_{\boldsymbol{z}, s^{\prime}}$, where

$$
\Omega_{\boldsymbol{z}, s^{\prime}}=\left\{\boldsymbol{\xi} \mid A_{12}\left(\omega, s, s^{\prime}, \omega, \boldsymbol{z}\right) \neq 0, \quad s \in\left[s_{a}, s_{b}\right]\right\} .
$$

Thus (13) gives a band-limited version of $R_{G}$ whose frequency content is given by $\cup_{s^{\prime}} \Omega_{\boldsymbol{z}, s^{\prime}}$. An edge of $R_{G}$ passing through $\boldsymbol{z}$ is visible if the normal of the edge lies in $\cup_{s^{\prime}} \Omega_{\boldsymbol{z}, s^{\prime}}$. In this regard (13) only reconstructs the visible edges of $R_{G}$. In the next section we will demonstrate the performance of our method in numerical simulations.

\section{NUMERICAL SIMULATIONS}

In our numerical simulations, we considered a scene of size $[0,22] \times[0,22] \mathrm{km}^{2}$ over a flat topography which is discretized by $128 \times 128$ pixels as illustrated in Figure 2; isotropic transmitter and receiver antennas; circular flight trajectories $\gamma_{R_{1}}(s)=\gamma_{R_{2}}(s+\pi / 4)=\gamma(s)=(11+11 \cos s, 11+11 \sin s, 6.5) \mathrm{km}$; uniform sampling for $s \in[0,2 \pi)$ at 512 points; and a transmitter located at $\mathbf{y}_{0}=(0,0,6.5) \mathrm{km}$ radiating a delta-like impulse. Thus we generated the projection data (see Figure 2) by substituting $A_{R_{i}}(\omega, s, \boldsymbol{x})=|\gamma(s)-\mathbf{x}|^{-1}$ and $A_{T}(\omega, s, \boldsymbol{x}, \mathbf{y})=\delta\left(\mathbf{y}-\mathbf{y}_{0}\right)|\mathbf{x}-\mathbf{y}|^{-1}$ in $(1):$

$$
d_{1, \mathbf{y}_{0}}(s, t)=d_{2, \mathbf{y}_{0}}(s, t)=d_{\mathbf{y}_{0}}(s, t)=\int \frac{\delta\left(t-\left[\left|\mathbf{y}_{0}-\mathbf{x}\right|+|\gamma(s)-\mathbf{x}|\right] / c_{0}\right)}{|\gamma(s)-\mathbf{x}|\left|\mathbf{x}-\mathbf{y}_{0}\right|} G(\boldsymbol{x}) d \boldsymbol{x} .
$$

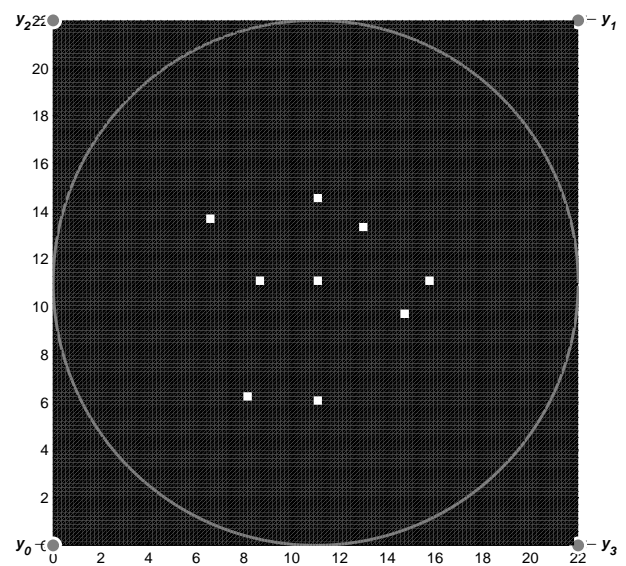

(a)

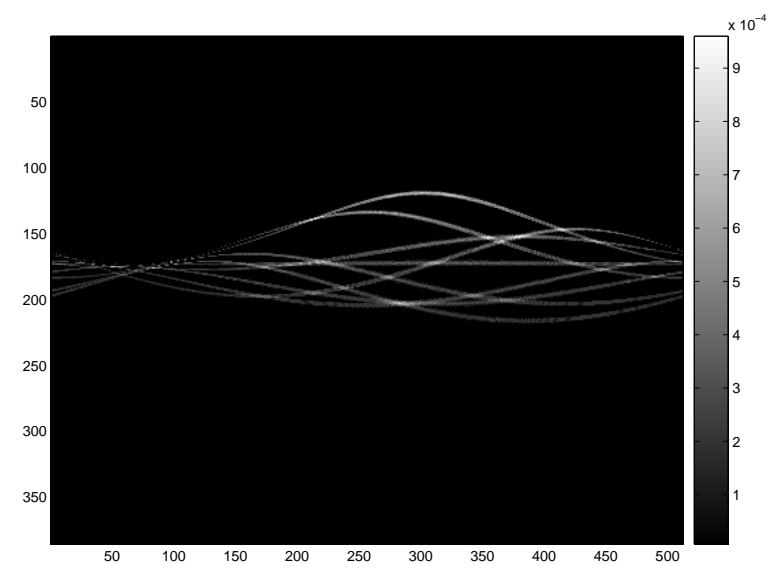

(b)

Figure 2. (a) $2 \mathrm{D}$ view of the scene reflectivity used in numerical simulations with transmitters located at $\mathbf{y}_{0}=(0,0,6.5) \mathrm{km}, \mathbf{y}_{1}=(22,22,6.5) \mathrm{km}, \mathbf{y}_{2}=(0,22,6.5) \mathrm{km}$, and $\mathbf{y}_{3}=(22,0,6.5) \mathrm{km}$, and the receiver trajectory $\gamma(s)=(11+11 \cos s, 11+11 \sin s, 6.5) \mathrm{km}$. (b) The projection data of the scene obtained for a single transmitter located at $\mathbf{y}_{0}$ and a receiver traversing $\gamma(s)$.

We assume that the scene is homogeneously illuminated by isotropic transmitters and set $\tilde{R}_{T}=1$. We present the corresponding reconstructed image in Figure 3. Due to the circular flight trajectories, all the edges of the target scene radiance are visible. Note that our choice of $\tilde{R}_{T}$ implicitly assumes that we have no information about transmitters' location. Therefore, the reconstructed images are not corrected with respect to the geometric spreading factor and the scatterers closer to the transmitter 


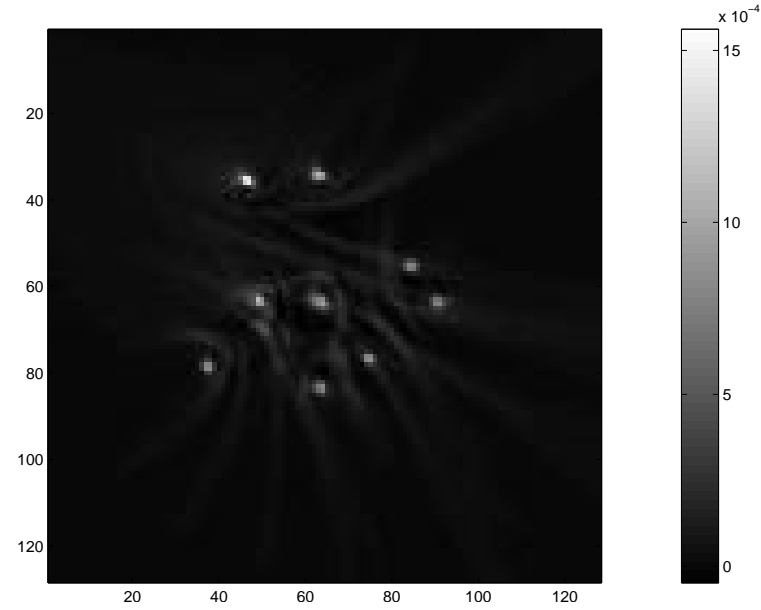

Figure 3. Reconstructed image by C-FBP for a single non-cooperative transmitter located at $\mathbf{y}_{0}$ and a receiver traversing $\gamma(s)$.

appear brighter than those that are farther away from the transmitter. However, when additional transmitters are placed equidistant from the scatterers this artifact is remedied as shown in Figure 4.b.

We considered four isotropic transmitters located at $\mathbf{y}_{0}=(0,0,6.5) \mathrm{km}, \mathbf{y}_{1}=(22,22,6.5) \mathrm{km}$, $\mathbf{y}_{2}=(0,22,6.5) \mathrm{km}$ and $\mathbf{y}_{3}=(22,0,6.5) \mathrm{km}$. We generated projection data $d$ by adding up the separately generated projection data for each of the receivers by

$$
d(s, t)=\sum_{i} d_{\mathbf{y}_{i}}(s, t) .
$$

Figure 4 shows the projection data and the corresponding reconstruction. The reconstructed scatterers that are closer to the transmitters are equally bright. The center of the scene, however, is illuminated with more energy than the single-transmitter case, thus the brightness level of the image is higher. This is due to the fact that the center of the scene is equidistant from the transmitters.

\section{CONCLUSION}

We presented a novel image reconstruction method, correlation-filtered backprojection (C-FBP), for a synthetic-aperture hitchhiker system in the presence of non-cooperative sources of opportunity. Our numerical simulations suggest that $\mathrm{C}-\mathrm{FBP}$ can be used in the presence of single and multiple transmitters.

The presented method can also be extended to operate in the presence of mobile sources of opportunity, clutter and noise. We will report on the performance of the proposed method for these cases in our future work.

\section{ACKNOWLEDGMENTS}

We are grateful to the Air Force Office of Scientific Research for supporting this work under the agreements FA9550-04-1-0223, and FA9550-06-1-0017. Because of this support, the U.S. Government is authorized to reproduce and distribute reprints for Governmental purposes notwithstanding any copyright notation thereon. The views and conclusions contained herein are those of the authors and should not be interpreted as necessarily representing the official policies or endorsements, either expressed or implied, of the Air Force Research Laboratory or the U.S. Government. 


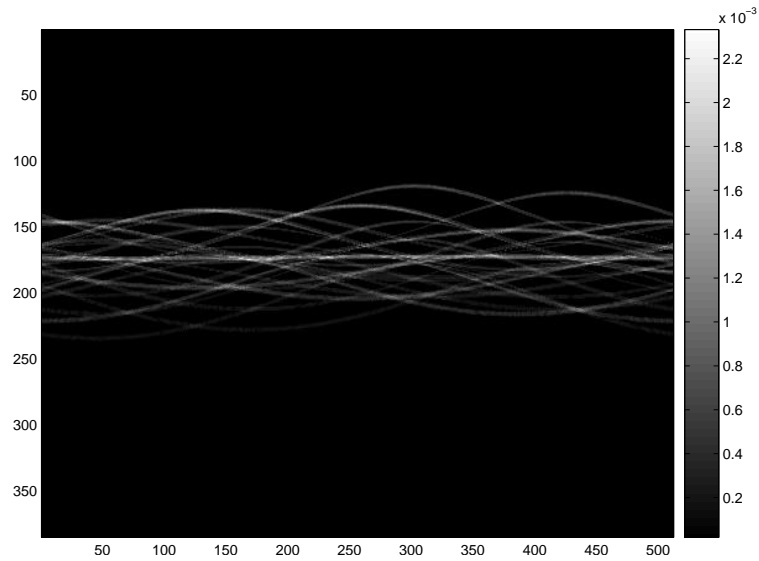

(a)

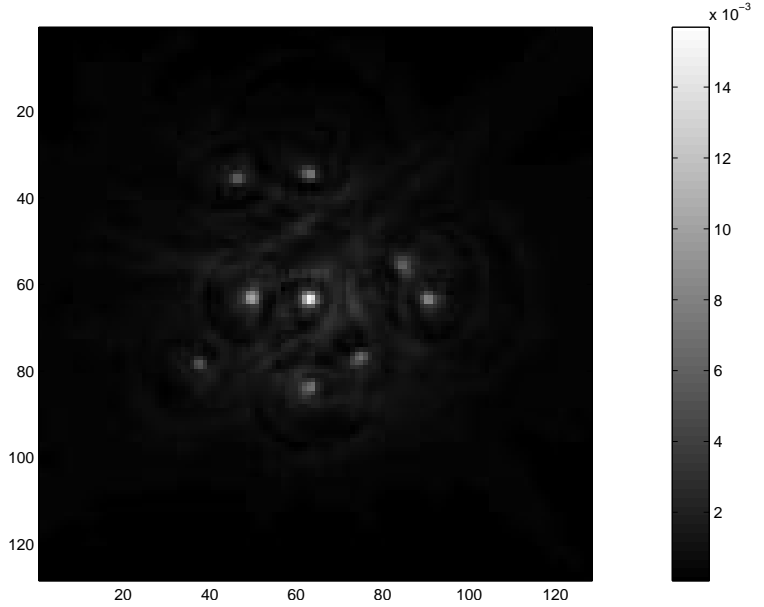

(b)

Figure 4. The projection data collected over a circular receiver trajectory $\gamma(s)$ due to (a) four transmitters located at $\mathbf{y}_{0}, \mathbf{y}_{1}, \mathbf{y}_{2}$ and $\mathbf{y}_{3}$ (see Figure 2), and (b) corresponding C-FBP reconstructed image.

\section{REFERENCES}

1. N. Willis, "Bistatic radar," in Radar Handbook, second edition, M. Skolnik, ed., ch. 25, McGraw-Hill, New York, 1990.

2. N. J. Willis, Bistatic Radar, Artech House, Norwood, MA, 1991.

3. Radar Systems Panel of the IEEE Aerospace and Electronic Systems Society, IEEE Standard Radar Definitions, 1997. IEEE Std 686-1997.

4. A. Horne and G. Yates, "Bistatic synthetic aperture radar," in IEEE RADAR 2002, pp. 6 - 10, Oct. 2002.

5. C. Yarman, B. Yazıcı, and M. Cheney, "Bistatic synthetic aperture hitchhiker imaging," in Proceedings of IEEE International Conference on Acoustics, Speech, and Signal Processing, April 2007.

6. H. Griffiths and N. Long, "Television-based bistatic radar," IEE Proc.-Radar Sonar Navig. 133(7), pp. 649$657,1986$.

7. P. Howland, D. Maksimiuk, and G. Reitsma, "FM radio based bistatic radar," 152, pp. 107-115, June 2005.

8. H. Griffiths and C. Baker, "Passive coherent location radar systems. part 1: Performance prediction," IEE Proc.-Radar Sonar Navig. 152, June 2005.

9. C. Baker, H. Griffiths, and I. Papoutsis, "Passive coherent location radar systems. part 2: Waveform properties," IEE Proc.-Radar Sonar Navig. 152, pp. 160-168, June 2005.

10. D. Poullin, "Passive detection using digital broadcasters (DAB, DVB) with COFDM modulation," IEE Proc.-Radar Sonar Navig. 152, pp. 143-152, June 2005.

11. D. Tan, H. Sun, Y. Lu, M. Lesturgie, and H. Chan, "Passive radar using global system for mobile communication signal: theory, implementation and measurements," IEE Proc.-Radar Sonar Navig. 152, pp. 116-123, June 2005.

12. "Roke Manor Research and FaberMaunsell Team to Drive Traffic Monitoring Technology Forward," http://www.roke.co.uk/press/46.php .

13. H. Griffiths, A. Garnett, C. Baker, and S. Keaveney, "Bistatic radar using satellite-borne illuminators of opportunity," in IEE Int. Conf. on Radar, pp. 276-279, October 1992.

14. V. Koch and R. Westphal, "New approach to a multistatic passive radar sensor for air/space defense," IEEE Aerospace and Electronic Systems Magazine 10, pp. 24-32, Nov. 1995.

15. M. Cherniakov, K. Kubik, and D. Nezlin, "Bistatic synthetic aperture radar with non-cooperative leos based transmitter," in Proceedings of IEEE International Geoscience and Remote Sensing Symposium, 2, pp. 861-862, July 2000. 
16. M. Cherniakov, K. Kubik, and D. Nezlin, "Radar sensors based on communication low earth orbiting satellites microwave emission," in Proceedings of IEEE International Geoscience and Remote Sensing Symposium, 3, pp. 1007-1008, July 2000.

17. J. Homer, K. Kubik, B. Mojarrabi, I. Longstaff, E. Donskoi, and M. Cherniakov, "Passive bistatic radar sensing with leos based transmitters," in Proceedings of IEEE International Geoscience and Remote Sensing Symposium, 2002.

18. M. Cherniakov, R. Saini, R. Zuo, and M. Antoniou, "Space surface bistatic sar with space-borne noncooperative transmitters," in Proceedings of European Radar Conference, pp. 25-28, Oct. 2005.

19. R. Pollard, "The role of passive radar sensors for air traffic control,"

20. H. Griffiths, "Radar imaging for combating terrorism," in NATO ASI 2006: Advances in Sensing with Security Applications, J. Byrnes, ed., NATO Security through Science Series.

21. X. He, M. Cherniakov, and T. Zeng, "Signal detectability in ss-bsar with gnss non-cooperative transmitter," IEE Proc.-Radar Sonar Navig. 152, pp. 124-132, June 2005.

22. I.-Y. Son, T. Varslot, C. E. Yarman, A. Pezeshki, B. Yazici, and M. Cheney, "Radar detection using sparsely distributed apertures in urban environment." to appear in SPIE Defense and Security Symposium, April 2007.

23. E. Attia and B. Steinberg, "Self-cohering large antenna arrays using the spatial correlation properties of radar clutter," IEEE Transactions on Antennas and Propagation 37, pp. 30-38, Jan. 1989.

24. R. J. Bonneau, "A rate distortion method for waveform design in rf target detection," in IEEE Aerospace Conference 2006, pp. 1-12, 2006.

25. M. Fink, W. Kuperman, J.-P. Montagner, and A. Tourin, eds., Imaging of Complex Media With Acoustic and Seismic Waves, Springer-Verlag, Berlin Heidelberg, 2002.

26. H. Barrett and K. Myers, Foundations of image science, Wiley-Interscience, Hoboken, NJ, 2004.

27. K. Wapenaar, D. Draganov, and J. Robertsson, Eds., "Seismic interferometry supplement," in Geophysics, 71, July-August 2006.

28. T.-K. Chan, Y. Kuga, and A. Ishimaru, "Experimental studies on circular SAR imaging in clutter using angular correlation function technique," IEEE Transactions on Geoscience and Remote Sensing 37, pp. 2192-2197, Sep. 1999.

29. C. J. Nolan and M. Cheney, "Synthetic aperture inversion," Inverse Problems 18, pp. 221-236, 2002.

30. C. Nolan and M. Cheney, "Synthetic aperture inversion for arbitrary flight paths and non-flat topography," IEEE Transactions on Image Processing 12, pp. 1035-1043, 2003.

31. C. Yarman, B. Yazici, and M. Cheney, "Bistatic synthetic aperture radar imaging for arbitrary flight trajectories and non-flat topography." to appear in IEEE Radar Conference, April 2007.

32. M. Soumekh, "Wide-bandwidth continuous-wave monostatic/bistatic synthetic aperture radar imaging," in Proceedings of International Conference on Image Processing, 3, pp. 361-365, Oct. 1998.

33. F. Treves, Introduction to Pseudodifferential and Fourier Integral Operators, volumes I and II, Plenum Press, NY, 1980. 